\title{
CARACTERIZACIÓN DE LA COMUNIDAD DE AVES EN EL PARQUE NACIONAL SIERRA MARTÍN GARCÍA, REPÚBLICA DOMINICANA
}

\author{
Hodali Almonte-Espinosa \\ Museo Nacional de Historia Natural "Prof. Eugenio de Jesús Marcano". \\ Calle César Nicolás Penson, Plaza de la Cultura Juan Pablo Duarte, Santo Domingo. \\ República Dominicana. h.almonte@mnhn.gov.do
}

\begin{abstract}
RESUMEN
Se presentan los resultados de una caracterización de la comunidad de aves del Parque Nacional Sierra Martín García, en una serie de puntos establecidos dentro de seis tipos de ambiente (bosque húmedo subtropical, bosque húmedo montano bajo, bosque de transición de zonas media, bosque seco, monte espinoso subtropical y llanura costera). Se registró un total de 1395 individuos, correspondientes a 90 especies y 37 familias. Del total de especies registradas, 14 son endémicas, 56 residentes, 18 migratorias y dos introducidas. El ambiente de llanura costera, presentó el más alto valor de riqueza de especies (68), mientras que el bosque húmedo montano bajo y el monte espinoso subtropical registraron los valores más bajos, cada uno con 24 especies. La mayor abundancia de individuos se registró en la llanura costera (448) y la menor en el bosque de transición en zona media (103). Los valores de diversidad de especies resultaron altos $\left(\mathrm{H}^{\prime}=3.9\right.$ y $\left.\mathrm{D}-1=0.97\right)$. El mayor valor de diversidad corresponde al ambiente costero $\left(\mathrm{H}^{\prime}=3.7\right.$ y $\left.\mathrm{D}-1=0.96\right)$, mientras que el bosque seco y el monte espinoso subtropical presentaron los valores más bajos cada uno con $\left(\mathrm{H}^{\prime}=2.9\right.$ y $\left.\mathrm{D}-1=0.93\right)$. De acuerdo con los criterios de la Unión Internacional para la Conservación de la Naturaleza (UICN), siete especies se encuentran amenazadas y dos casi amenazadas.
\end{abstract}

Palabras clave: riqueza de especies, diversidad, abundancia, especies amenazadas.

\section{ABSTRACT}

The results of a characterization of the bird community of Sierra Martín García National Park are presented in a series of points established within six types of environments (humid subtropical forest, low montane humid forest, middle area transition forest, dry forest, lowland subtropical thorn scrub and coastal plain). A total of 1395 individuals and 90 species were recorded in 37 families of birds. Of the total number of species recorded, 14 are endemic, 56 resident, 18 migratory and two introduce. Coastal plain presented the highest value of species richness (68), while the humid forest and the subtropical thorn scrub recorded the lowest values, each with 24 species. The greatest abundance of individuals was documented by the coastal plain (448) and the smaller by the transition forest (103). The bird community of this protected area presents a high species diversity $\left(\mathrm{H}^{\prime}=3.9\right.$ and $\left.\mathrm{D}=0.97\right)$. The coastal plain showed the highest diversity value $\left(H^{\prime}=3.7\right.$ and $\left.\mathrm{D}=0.96\right)$. The dry forest and the lowland subtropical thorn scrub had the lowest diversity value $\left(H^{\prime}=2.9\right.$ and $\left.\mathrm{D}=0.93\right)$. According to the International Union for Conservation of Nature (IUCN) criteria, seven species are considered threatened and two nearly threatened. 


\section{INTRODUCCIÓN}

El Parque Nacional Sierra Martín García constituye uno de los lugares con mayor diversidad de aves en la Hispaniola, siendo considerada como unas de las áreas de importancia para la conservación de las aves (AICA; Perdomo et al., 2010). La gran diversidad de ambientes presentes en la zona favorece la existencia de una amplia gama de grupos de aves, tanto terrestres como acuáticas, encontrándose distribuidas en lagunas, playas, canales y llanos del área costera, así como en bosques densos, áreas deforestadas y zonas con vegetación arbustiva.

En el área han sido reportadas 105 especies de aves, 21 de éstas endémicas (Perdomo et al., 2010), entre ellas el Gavilán de la Hispaniola (Buteo ridwayi), la cual fue extirpada de la zona y actualmente está confinada al Parque Nacional Los Haitises (Birdlife International y Grupo Jaragua, 2005).

El presente trabajo analiza las comunidades de aves en seis tipos de ambiente del Parque Nacional Sierra Martín García, contribuyendo así al conocimiento sobre la composición, riqueza, diversidad, abundancia y estado de conservación del grupo en la zona de estudio.

\section{OBJETIVO}

-Caracterizar y comparar las comunidades de aves en seis tipos de ambiente del Parque Nacional Sierra Martín García.

\section{MATERIALES Y MÉTODOS}

Área de estudio. La Sierra Martín García (SMG) es un macizo montañoso de relieve accidentado ubicado entre las provincias de Azua y Barahona, frente a la Bahía de Neiba, en el suroeste de la República Dominicana. Sus puntos más elevados son Loma del Curro $(1350 \mathrm{~m})$ y Loma Fría, $1285 \mathrm{~m}$ (SEA/DED, 1990; Perdomo et al., 2010). Según SEA/DED (1990), entre los ambientes presentes en esta área protegida están: bosque húmedo subtropical (BHS), bosque húmedo montano bajo (BHM), bosque de transición de zonas medias y bajas (BTM), bosque seco (BS), monte espinoso subtropical (MES), sabana de halófitos (SH), dunas (DU) y llanuras costera (LC).

Las localidades muestreadas según el tipo de ambiente fueron: Loma del Curro, bosque húmedo subtropical; Cañada Azul, bosque húmedo montano bajo; Montilla Arriba, bosque de transición de zonas medias; Montilla Abajo, bosque seco; Montilla Abajo, monte espinoso subtropical y Puerto Alejandro, llanura costera. En este trabajo se consideró como llanura costera (LC) al área de Puerto Alejandro y alrededores, la cual incluye diferentes playas, lagunas, canales, manglares, marismas y el bosque seco próximo a este.

Metodología. Se utilizaron diferentes métodos de muestreo para evaluar la riqueza de especies. Se establecieron 60 puntos de conteo distribuidos de manera heterogénea en siete transectos. Los puntos de conteo estuvieron separados entre sí por una distancia mínima de aproximadamente $200 \mathrm{~m}$. En la llanura costera se hicieron dos transectos, cada uno de $1 \mathrm{~km}$. Solo se identificaron las especies que se encontraban en un perímetro limitado de $50 \mathrm{~m}$ de radio (Ralph et al., 1996). Los datos fueron tomados en intervalos de diez minutos. Se identificaron todas las especies observadas y escuchadas. Luego de terminar con la metodología anterior se procedió a utilizar el método de registro no sistemático de aves, el cual consiste en realizar recorridos aleatorios dentro del área de interés para detectar el mayor 
número de especies posible (Fjeldsa, 1999). La ventaja de este es que las observaciones pueden realizarse durante todo el día, además, pueden incluirse especies que no fueron reconocidas de forma sistemática.

Los horarios establecidos para la realización de los conteos fueron desde 7:00 hasta las 10:00 horas y desde las 16:00 hasta las 18:00 horas. Las observaciones se realizaron con binoculares Nikon 8x42. Para la identificación y ordenamiento taxonómico se siguió a Latta et al., 2006, y para la actualizaciones de los táxones se consideraron diferentes suplementos de The American Ornithologists Union, AOU (Banks et al., 2005, 2006, 2007, 2008; Chesser et al., 2009, 2010, 2014, 2017).

Diversidad. Con la finalidad de conocer mejor las comunidades en estudio, se usaron varios índices de diversidad y equidad de especies: índice de diversidad de Shannon ( $\left.H^{\prime}\right)$, índice de equidad de Pielou (J'), índice de dominancia de Simpson ( $\lambda$ ), recíproco de Simpson (D-1), siempre de acuerdo a Moreno (2001).

\section{RESULTADOS Y DISCUSIÓN}

Riqueza y abundancia. Se registraran 1395 individuos y 90 especies de aves, distribuidas en 37 familias. De estas 14 son endémicas, 56 residentes, 18 migratorias y dos introducidas (Tabla I). El número de especies reportadas en este trabajo, difiere de las 105 listadas por Perdomo et al. (2010) y las 67 encontradas en la evaluación de los recursos naturales en la Sierra Martín García y Bahía de Neiba (SEA/DED, 1990). Es posible que en el primer caso esta discrepancia se deba a que en el presente trabajo no fueron muestreadas las áreas próximas a la comunidad de Barrera, provincia Azua. Además, el actual estudio fue realizado al inicio y al final de la temporada de migración, debido a esto las especies que migran entre finales de septiembre-noviembre y marzo-abril no quedarían registradas.

Igualmente, se encontraron diferencias en el número de especies endémicas reportadas, siendo menor a las 21 registradas por Perdomo et al. (2010). Entre las especies que no fueron registradas: el Chirrí (Calyptophilus frugivorus), la Cigüita Aliblanca (Xenoligea montana), el Papagayo (Priotelus roseigaster) y el Cao (Corvus palmarum).

Se hace el primer registro para República Dominicana de la especie migratoria Barga Jaspeada (Limosa fedoa), familia Scolopacidae, de la cual solo existían dos reportes para la Hispaniola (1961 y 1963), ambos realizados en Haití (Latta et al., 2006).

En los seis ambientes estudiados, las especies más comunes fueron: la Rolita (Columbina passerina), el Ruiseñor (Mimus polyglottos), el Barrancolí (Todus subulatus), el Gallito Prieto (Loxigilla violacea) y el Cuatro Ojos (Phaenicophilus palmarum). Todas con un amplio rango de distribución y consideradas como comunes y abundantes a lo largo de la isla.

La riqueza de especies varió de 24 a 68 por tipo de ambiente. Las zonas de mayor riqueza fueron la llanura costera (LC) y el bosque húmedo subtropical (BHS), con 68 y 44 especies, respectivamente; mientras que el bosque húmedo montano (BHM) y el monte espinoso subtropical (MES) presentaron la menor, ambos con 24 (Tabla I). La mayor abundancia se obtuvo en la llanura costera con 448 individuos, mientras que el bosque de transición de áreas medias presentó la menor con 103 individuos (Tablas I y II). 


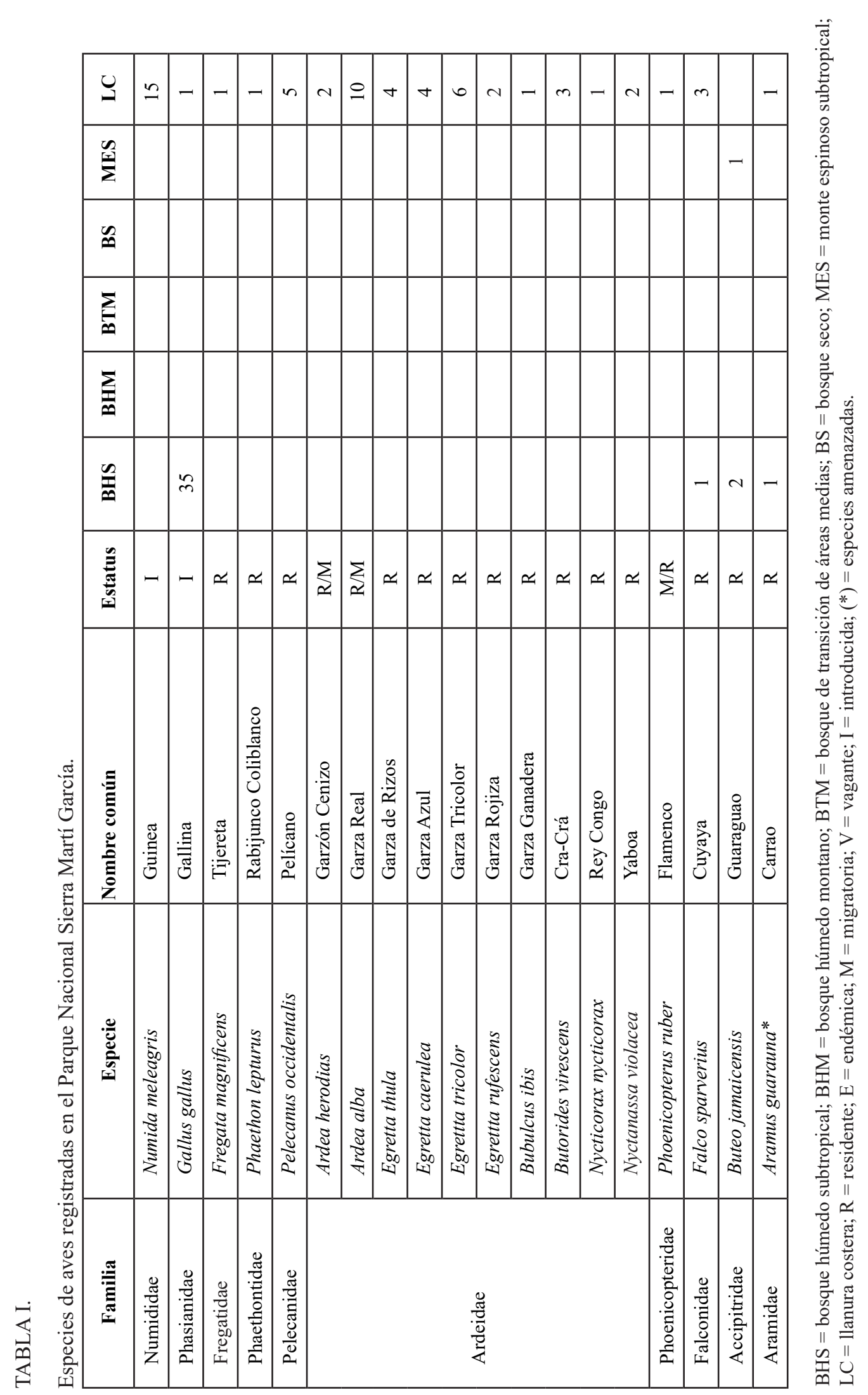




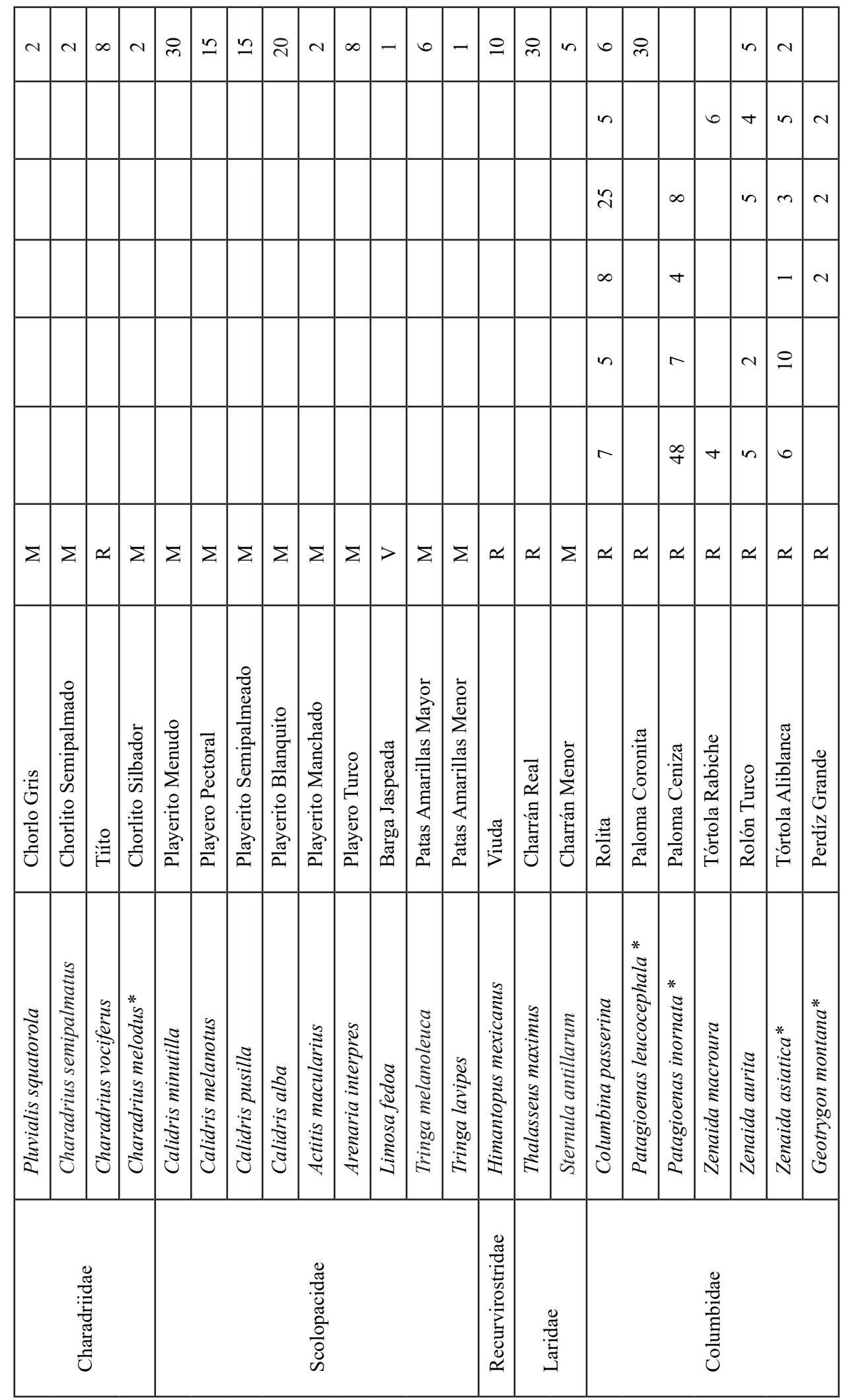









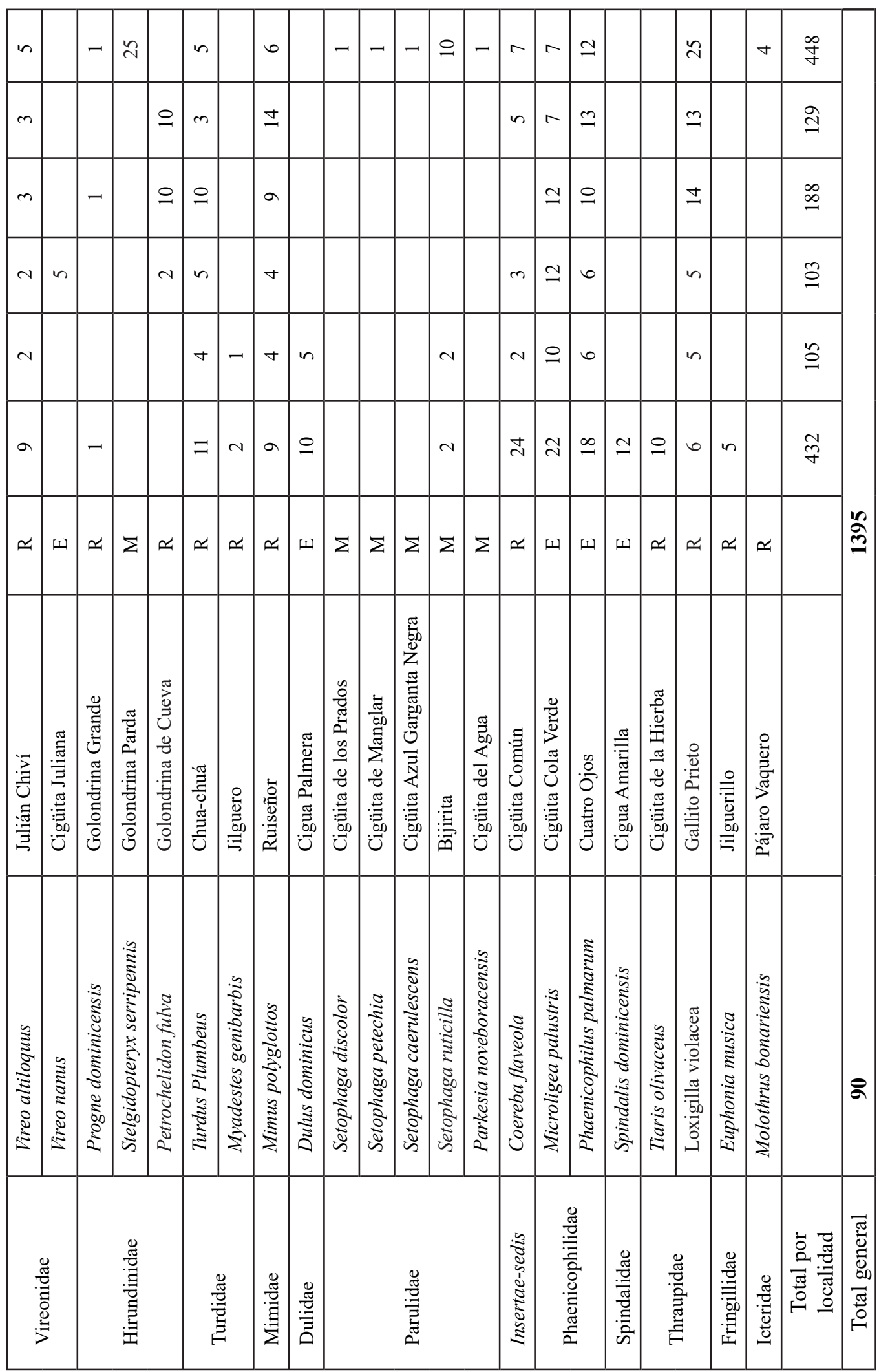


Los valores de riqueza y abundancia de especies registrados en LC, podrían estar relacionados con la multiplicidad de ambientes presentes en el área de Puerto Alejandro y sus alrededores: playas, lagunas, canales, manglares y la vegetación de bosque seco costero. Además, esta zona podría servir como punto de congregación de aves migratorias, lo cual explicaría la presencia de algunas especies a principio de septiembre, tales como la Bijirita (Setophaga ruticilla), la Cigüita de los Prados (Setophaga discolor) y una gran variedad de aves acuáticas y costeras como; el Playerito menudo (Calidris minutilla), el Chorlito Semipalmado (Charadrius semipalmatus) y la Barga Jaspeada (Limosa fedoa), entre otras.

Diversidad. Los resultados obtenidos con el índice de diversidad de Shannon y el recíproco de Simpson (para los seis tipo de ambiente en conjunto), sugieren una alta diversidad de aves $\left(\mathrm{H}^{\prime}=3.9\right.$ y $\left.\mathrm{D}-1=0.97\right)$. Probablemente estos resultados se relacionen con la gran variedad de ecosistemas presentes y el buen estado de conservación de algunas de las áreas muestreadas (bosque seco y monte espinoso subtropical).

TABLA II.

Resumen de datos ecológicos de las áreas en estudio.

\begin{tabular}{|c|c|c|c|c|c|c|c|}
\hline & BHS & BHM & BTM & BS & MES & LC & SMG \\
\hline S & 44 & 24 & 26 & 25 & 24 & 68 & 90 \\
\hline$H^{\prime}$ & 3.12 & 3.11 & 2.92 & 2.90 & 2.90 & 3.7 & 3.92 \\
\hline $\mathrm{J}^{\prime}$ & 0.82 & 0.94 & 0.92 & 0.89 & 0.91 & 0.88 & 0.87 \\
\hline$\lambda$ & 0.05 & 0.05 & 0.08 & 0.07 & 0.07 & 0.04 & 0.03 \\
\hline D-1 & 0.95 & 0.95 & 0.93 & 0.93 & 0.93 & 0.96 & 0.97 \\
\hline Abundancia & 432 & 105 & 103 & 188 & 129 & 448 & 1395 \\
\hline
\end{tabular}

Índice de diversidad de Shannon (H'), índice de equidad de Pielou (J'), índice de dominancia de Simpson $(\lambda)$ y recíproco de Simpson (D-1). BHS = bosque húmedo subtropical, BHM = bosque húmedo montano, BTM = bosque de transición de áreas medias, $\mathrm{BS}=$ bosque seco, $\mathrm{MES}=$ monte espinoso subtropical y $\mathrm{LC}=$ llanura costera.

De acuerdo con el índice de Shannon, estos ecosistemas presentan una diversidad alta; registrándose ligeras variaciones entre las comunidades evaluadas. La llanura costera (LC) mostró el mayor valor $\left(\mathrm{H}^{\prime}=3.7\right.$; Tabla II). Igualmente, presentó el valor más bajo de dominancia de especies $(\lambda=0.04)$; siendo su recíproco $(\mathrm{D}-1=0.96)$. Al comparar este ambiente con los demás se encontró una mayor variedad de ecosistemas tanto acuáticos como terrestres; lo cual probablemente, favorece una gran diversidad de grupos de aves (Tabla II).

Los resultados obtenidos con el índice de equidad de Pielou, tanto para el área en conjunto como por tipo ambiente, sugieren que en los ecosistemas evaluados las comunidades de aves se distribuyen de forma homogénea (Tabla II). Esto se refleja en los índices de diversidad utilizados ya que, por lo general, a mayor equidad mayor diversidad de especies.

Especies amenazadas. De acuerdo con los criterios de la Unión Internacional para la Conservación de la Naturaleza (UICN), siete especies se encuentran amenazadas: el Carrao (Aramus guarauna), el Chorlito Silbador (Charadrius melodus), la Perdíz Colorada (Geotrygon montana), la Cotorra (Amazona ventralis) el Perico (Psittacara chloropterus), la Paloma Coronita (Patagioenas leucocephala) y la Paloma Ceniza (Patagioenas inornata); y dos casi amenazadas: Tórtola Aliblanca (Zenaida asiatica) y el Torico (Siphonorhis brewsteri). Se confirmó que la destrucción y fragmentación de los hábitats, la cacería indiscriminada y el pichoneo, son factores que inciden sobre las poblaciones de aves del lugar. 


\section{AGRADECIMIENTOS}

Al Museo Nacional de Historia Natural "Prof. Eugenio de Jesús Marcano" por el auspicio de este trabajo. A mis compañeros de campo Francisco Paz, Katihusca Rodríguez, Patricia Torres y Cristian Marte. Agradecimientos especiales a Bertho Díaz, quien fue de gran ayuda durante el trabajo de campo. A Carlos Suriel, Miguel Núñez, Gabriel de los Santos y Miguel Landestoy por sus correcciones y sugerencias.

\section{LITERATURA CITADA}

Banks, R., K. Barker, C. Cicero, J. Dunn, A. Kratter, P. Rasmussen, J. Remsen, J. Rising y D. Stotz. 2005. Forty-Sixth Supplement to the American Ornithologists' Union Check-List of North American Birds. The American Ornithologists' Union. The Auk, 122 (3): 1026-1031.

Banks, R., C. Cicero, J. Dunn, A. Kratter, P. Rasmussen, J. Remsen, J. Rising y D. Stotz. 2006. Forty-Seventh Supplement to the American Ornithologists' Union Check-List of North American Birds. The Auk, 123 (3): 926-936.

Banks, R., K. Barker, C. Cicero, J. Dunn, A. Kratter, P. Rasmussen, J. Remsen, J. Rising y D. Stotz. 2007. Forty-Eighth Supplement to the American Ornithologists' Union CheckList of North American Birds. The Auk, 124 (3): 1109-1115.

Banks, R., T. Chesser, C. Cicero,J. Dunn, A. Kratter, I. Lovette, P. Rasmussen, J. Remsen, J. Rising, D. Stotz, y K. Winker. 2008. Forty-Ninth Supplement to the American Ornithologists' Union Check-list of North American Birds. The American Ornithologists' Union. The Auk, 125 (3): 758-768.

Birdlife International y Grupo Jaragua, 2005. http://datazone.birdlife.org/site/factsheet/sierramartin-garcia-iba-dominican-republic. Consultado 27-XII-2016.

Chesser, T., R. Banks, C. Cicero, J. Dunn, A. Kratter, P. Rasmussen, I. Lovette, J. Remsen, J. Rising, D. Stotz y K. Winker. 2009. Fiftieth Supplement to the American Ornithologists' Union Check-list of North American Birds. The American Ornithologists' Union. The Auk, 126 (3): 705-714.

Chesser, T., R. Banks, K. Barker, C. Cicero, J. Dunn, A. Kratter, I. Lovette, P. Rasmussen, J. Remsen, J. Rising, D. Stotz, y K. Winker. 2010. Fifty-First Supplement to the American Ornithologists' Union Check-List of North American Birds. The American Ornithologists' Union. The Auk, 127 (3): 726-744.

Chesser, T., R. Banks, C. Cicero, J. Dunn, A. Kratter, I. Lovette, A. Navarro, P. Rasmussen, J. Remsen, J. Rising, D. Stotz y K Winker. 2014. Fifty-Fifth Supplement to the American Ornithologists' Union Check-List of North American Birds. The Auk, 131: CSi-CSxv.

Chesser, T., K. Burns, C. Cicero, J. Dunn, A. Kratter, I. Lovette, A. Navarro, P. Rasmussen, J. Remsen, J. Rising, D. Stotz y K Winker. 2017. Fifty-eighth supplement to the American Ornithologists 'Union Check-List of North American Birds. The Auk, 134: 751-773.

Fjeldsa, J. 1999. The impact of human forest disturbance on the endemic avifauna of the Udzungwa Mountains, Tanzania. Bird Conservation International, 9 (10): 47-62. 
Latta, S., C. Rimmer, A. Keith, J. Wiley, H. Raffaele, K. Mcfarland y E. Fernández. 2006. Aves de la República Dominicana y Haití. Princeton University Press. VII+258 pp.

Moreno, C. E. 2001. Método para medir la biodiversidad. M\&T-Manuales y Tesis SEA, vol.1. Zaragoza, 84 pp.

Perdomo, L., Y. Arias, Y. Léon y D. Wege. 2010. Áreas importantes para la conservación de las aves en la República Dominicana. Grupo Jaragua y el programa IBA-Caribe de Birdlife International: República Dominicana. Santo Domingo, República Dominicana, 84 pp.

Ralph, C. J., G. R. Geupel, P. Pyle, T. E. Martín, D. F. DeSante y B. Milá. 1996. Manual de métodos de campo para el monitoreo de aves terrestres. Gen. Tech. Rep. PSW-GTR-159. Albany, CA: Pacific Southwest Research Station, Forest Services, U. S. Department of Agriculture, $46 \mathrm{pp}$.

SEA/DED. 1990. Evaluación de los recursos naturales en la Sierra Martín García y Bahía de Neiba. Secretaría de Estado de Agricultura y Servicio Alemán de Cooperación SocialTécnica. Santo Domingo, República Dominicana, 80 pp. 